\title{
Author Index Vol. 19,1996
}

Autorenverzeichnis Band 19,1996/

ONKOLOGIE

Alken,P. 187,189 Altwein,J.E. 4302 Arendt, T. 5410 Arps,H. 4334

Balitrand,N. SI 10 Bamberg,M. 6508 Bankmann, Y. 6490 Baumgart, J. 2153 Beck,W.T. SI 14 Berdel,W.E. 4 328,6 490 Beuth,J. 2182 Bex, A. 131 Bischoff,S. 5424 Boehme,V. 5410

Boerde,E.C 6480 Böhning,K. 5430 Bornemann, K. 5424 Bremer, G. 2170 Bremer,K. 2170

Broen von, I.-M. 6490 Buhr,H. 3214 Burg, G. 3226 Busch,M. 5 430,6 501 Bültmann,B. 6508

Castaigne, S. SI 10 Chomienne, Ch. SI 10 Clemens M.R. 162 Comely, D. 6506 Cornic, M. SI 10

Danks,M.K. SI 14

Debatin, K.-M. SI 3

Debois,J.M. 5385

Degner,C. 6506

Degos,L. SI 10

Delva,L. SI 10

Depisch,D. 2147

Diezler,P. 162

Dimitrakopoulou-Strauss, A. 3253

Djavanmard, M. 3242

Doll,J. 3253

Drings,P. 3248

Dühmke,E. 5 430,6 501

Dummer, R. 3226

Eberhard, A. 2170

Eberhardt, W. 2153

Eckersberger, F. 3221

Edler, L. 1 62, 3 248, 4 328, 5404

Eigler,F.W. 5416

Eil,A. 3253

Engler,H. 2126

Faiss,S. 2119,3 214 Fiebig,H.H. 3 248,4 328 Fink,U. 5416 Fölsch,U.R. 5410 Franke,T. 6501 Frank, St. 116 Fritze,D. 162

Gabius,H.-J. 174 Gabius,S. 174 Gadner,H. 3234 Gatzemeier, U. 3 248, 4328 Gawlik, C. 4334 Georgi,M. 4337 Gerhard, M. 2181 Gnant,M. 3242 Goepel,M. 124,131 Götzinger, P. 3242 Graf,E. 2184 Graf,N. 136 Gramlich, D. 4364

Häffner,A.C. 3226 Hagmüller, E. 162 Hanauske, A.-R. 5404 Härle,M. 162 Harstrick,A. 2153 Hartenstein, R. 5419 Hartmann, F. 2114 Hartung, G. 162,184 Hastka,J. 5437 Haustrate,FM. 5 385 Haverkamp, U. 2192 Hehlmann,R. 144,5 437 Heidemann, E. 2140 Hejna,M. 2164 Hellriegel,K.P. 194 Hennemann, H.H. 3266 Henß,H. 4328 Herbst,K. 6490 Hess, C.F. 5444 Hess, M. 3226 Hieber,U. 182 Hill,B.T. SI 6 Hochhaus, A. 144 Hoffknecht, M. 162 Hoffmann, 
W. 6508 Holstein,K. 6496 Horng,M. SI 19 Hossfeld, D. K. 2 159, 6 490, 6496 Hörmann, K. 1 81 Hörmann, R. 4342 Huber,H. 3 209,3 221

Illhardt, FJ. 2187

Jacob, I. 162 Jakesz, R. 3242 Jakse, G. 4296 Jiao,L. SI 19 Jockenhövel, 4348 Juhl, H. 2181

Jung, P. 4296 Jungius, K.-P. 4337

Käfer, G. 5437 Kandioler,D. 3242 Karall, M. 2147 Kashani-Sabet, M. SI 19 Kashfian,B.L SI 19 Katz,R. 162 Kirsch,K. 168 Klaassen, U. 2153 Kleeberg, U. R. 4 334, 5446 Kiestil,Th. 154 Köhrmann,K.U. 181 Korfel,A. 6490 Korn,M. 2153,5 416 Kornek,GV. 2147,2164 Kovar,H. 3 234 Krajnik, G 3221 Krege,S. 124 Krismer, M. 154 Kudielka,R.A. 4315 Kurth,K. 6480 Laurent, D. 5444 Lefebvre,P. SI 10 Lehnert, M. 6474 Lengfelder, E. 144 Lorenz, G 5410 Ludwig, H. 2164 Lüftner, D. 4308

Manegold, C. 182, 3 248, 4328 Mann,K. 4340 Marian,B. 2132 Mergenthaler, H.-G 4308 Meyer, H.-J. 5424 Micus, S. 2170 Milch,W. 168 Mitrou,P.S. 149 Möhr,T. 3260 Mross,K. 6 490 Müller,M.J. 5424 Müller-Wieland, D. 4348 Münstedt, K. 168

Neises, M. 191 Neumeier, M. 2181

Oberdorfer, F. 3253 Ofner, H. 2192 Osieka,R. SIV,Sll Otto,T. 124,131

Panzer, M. 5419 Parwaresch, R. 6464 Paul, D.R. 4334 Pawel, J. v. 3248 Pfreundschuh, M. 2114 Pirker, R. 3221 Possinger, K. 4308 Potter, R. 2192 Pradier, O. 5444 Preiss, R. 2176 Queißer, U. 4337 Queißer, W. 162,1 82, 3 209, 3 248, 3 260, 4 337, 4 354, 5437

Raab, R. 5424 Rachbauer, F. 154 Ranft,K. 5440 Rave-Fränk, M. 5 430, 6501 Reijkede,T.M. 6 480 Reimer, Ch. 168 Renner, C. 2114 Renner, U. 4340 Resch-Holeczke, A. 2192 Reuter, H. 5 440 Riecken, E.-O. 2119,3 214 Riesen,W.F. 2126 Rieth,A. 4315 Rohrbacher, M. 2147

Rübben,H. 124,131 Rudas, M. 3242 Rudolph, P. 6464 Runkel,N. 6506

Sachsse, S. 168 Salem, G. 2147 Sailer, B. 4350

Salzer,G.M. 3221 Sauer, O. 185 Sauerbrei,W. 2184 Scanlon,K.J. SI 19 Schackert, G. 16,116 Schackert,H.K. 116 Schamhart,D.H.J. 6480 Scheithauer, W. 2 147, 2164 Schenk,T. 2164 Scherübl,H. 2119,3 214 Scheulen,M.E. 2153 Schiebe,M. 6508 Schirren,J. 2290 Schlag,PM. 3 208,5 394 Schmid,A. 5410 Schmidberger, H. 5444 Schmidt, R 2176 Schmoor, C. 178

Schneider, P. 4290 Schönfelder, M. 2176 Schulz,K.D. 194 Schumacher, M. 178,2184 Schuth,J. 4315 Seeber,S. 2153,5 416 Seifert,A. 162 Seifert,V. 4346 Selberg, O. 5424 Siegsmund,M.

187,189 Siewert,J.R. 5416 Silchinger, J. 4302 Sorger,D. 2176 Stahl,M. 175,3 211,5 416 Stalla, GK. 4340 Steger, G 3242 Stegmaier, C. 3268 Steinmetz, A. 16 Stenson,K.M. 5399 Sterzinger, W. 154 Stoffregen, C. 5410 Stolke, D. 4340 Strauss, L.G. 3253 Strittmatter, H.J. 192

Strumberg D. 2153 Study Group Colon and Rectum Carcinoma 162

Taucher, S. 3242 Terry,N.H.A. 4322 Thürlimann, B. 2126 Tilgen,W. 3253 Trainer, S. 4290 Unbehaun, V. 2189

Vahrson,H. 168 Vanhoefer,U. 5416 Versteeg, R. 4334 Vogel,U. 6508 Vogt-Moykopf, I. 4290 Vokes,E.E. 5399 von Pawel, I. 4328

Walter, F. 2176

Walter, T. A. 6496

Wassner, A. 2140

Weber, A. 187,189

Weh, H.-J. 162, 2 159,3 266, 6496

Weimann, A. 5424

Weirich,A. 136

Weiss, H. 162 
Wendt,T.G 5419

Wiedenmann, B. 2 119, 3214

Wiegel,T. 6506

Wilke,H. 2153,3 211,5 416

Wilier, A. 5437

Wit, M. 2159

Wojatscheck, C. 162

Wolverton, J.S. SI 14

Wustrow, T.P.U. 5419

Xia,L. SI 19

Ziegler,H. 3268 Zimmer,T. 2119,3 214 Zöchbauer, S. 3221 Zornig,C 2159 Zoubek,A. 3234

Zurborn, K.-H. 5410

528

Autorenverzeichnis Onkologie, Band 19,1996 Author Index Onkologie, Vol. 19,1996 